\title{
Parámetros de calidad de imagen de tomosíntesis mamaria: Estudios con fantomas antropomórficos deformables
}

\section{Image quality metrics of breast tomosyntesis: Studies with deformable anthropomorphic phantoms}

Presentación: 6-7/10/2020

\section{Doctorando:}

\section{Rosana Pichio}

Comisión Nacional de Energía Atómica, Física Médica, Facultad Regional de Buenos Aires, Universidad Tecnológica Nacional Argentina

rosanapirchio@cnea.gob.ar

\section{Director/a:}

\section{Claudio Delrieux}

\section{Co-director/a:}

\section{Eduardo E. Rodríguez}

\section{Resumen}

Tomosíntesis digital de mama (DBT) es una tecnología relativamente novedosa y para realizar controles de calidad de las imágenes obtenidas por ese equipo, se requieren fantomas específicos de 3 dimensiones que simulen lo mejor posible las mamas de las mujeres. El objetivo de este trabajo es diseñar fantomas virtuales (in-silico) en base a imágenes obtenidas con un sistema DBT , para utilizarlos en la fabricación de fantomas antropomórficos de mama con materiales elastoméricos tales como polivinil alcohol y silicona. También se adquirirán imágenes DBT para entrenar un modelo de análisis basado en el aprendizaje de máquina. La calidad de los fantomas construidos se validarán a partir de imágenes mamográficas. Finalmente, se realizarán pruebas de fuerza de compresión y calidad de imágenes empleando los nuevos fantomas.

Palabras clave: Tomosíntesis, mamografía, fantomas antropomórficos, fuerza compresión.

\begin{abstract}
Digital Breast Tomosynthesis (DBT) is a relatively new technology and in order to perform quality controls of the images obtained by that equipment, specific 3 dimensional phantoms are required that simulate a woman breast the best as possible. The objective of this work is to design virtual phantoms (in-silico) based on images obtained from DBT system to use them in the manufacture of anthropomorphic breast phantoms with elastomeric materials such as polyvinil alcohol and silicone. DBT images will also be acquired to train a analysis model based on machine learning. The quality of phantoms will be validated from mammographic images. Finally, compression forces and image quality tests will be performed with the new phantoms.
\end{abstract}

Keywords: Tomosynthesis, mammography, anthropomorphic phantom, compression force 


\section{Introducción}

El cáncer de mama es la primera causa de muerte de mujeres en Argentina, seguido por el cáncer de pulmón. De acuerdo al Proyecto GLOBOCAN 2018 se diagnostican 21558 nuevos casos por año y mueren por esta neoplasia 6380 mujeres (IARC, 2020) . En tanto, la Organización Mundial de la Salud afirma que el cáncer de mama es el segundo cáncer más común en el mundo y es la segunda causa de muerte de las mujeres de los países del primer mundo (WHO, 2020).

La principal herramienta para la detección precoz del cáncer de mama es la mamografía, la cual ha logrado disminuir hasta un 30\% la mortalidad de las pacientes al lograr la detección de lesiones de apenas 5 milímetros de diámetro; con una sensibilidad de 75/90\% y una especificidad del 90/95\% (INC, 2020).Los casos que presentan mayores complicaciones en este tema corresponden a pacientes jóvenes, con mamas heterogéneamente densas, para las que se sabe que son más propensas a padecer cáncer de mama y es más complejo su diagnóstico.

La tomosíntesis digital de mama es un sistema de rayos $\mathrm{X}$ que emergió en el mercado hace aproximadamente una década y es básicamente una mamografía digital cuasi 3D. En esta técnica el detector está fijo y el cabezal gira alrededor de un centro de rotación ubicado generalmente en la parte superior de la mama. El plano de giro es perpendicular a la dirección ánodo-filtro; el arco descripto y la cantidad de proyecciones son pequeños, y los valores de dosis media glandular en cada proyección son bajos (EUREF; 2016).

De acuerdo a Basic Safety Standards,es necesaria una adecuada calidad de imágenes y que la dosis media glandular sea lo más baja posible para asegurar un correcto diagnóstico de los estudios mamográficos. Esto involucra la realización de prácticas de controles de calidad y de optimización (IAEA, 2014).

Los protocolos de control de imágenes para DBT (EUREF, 2016) mencionan el empleo de determinados fantomas que simulan una mama. En el mercado hay una gran variedad de fantomas: fantoma de acreditación del American College of Radiology (ACR), CDMAM, NORMI PAS (NORMI PAS, 2013), de entrenamiento para biopsia (CIRS, 2020), entre otros, y para la simulación 3D: TMIST, BR3D-CIRS (CIRSINC; ARTINIS, 2020)

Sin embargo, debido a la nueva tecnología de los equipos DBT, es necesario utilizar fantomas antropomórficos. Para ello se desarrollaron fantomas con PVAL (Price, 2010; Akki et al., 2014; Hossain et al., 2015), aceites (Feng et al., 2012), siliconas (Silva et al., 2012; Daskalaki et al., 2016 ) y con materiales que se pueden aplicar mediante impresión 3D (Schopphoven et al., 2019; Kiarashi et al., 2015) . En los últimos años se enfatizó la necesidad de crear fantomas antropomórficos. En Europa se comenzaron a desarrollar fantomas virtuales (Baneva et al., 2016; Bliznakova et al., 2009) y existen algunos desarrollos de fantomas físicos (Feradov et al., 2019; Bliznakova et al., 2018) .

Un objetivo importante es lograr que las imágenes de los fantomas, así como las imágenes de los/las pacientes, puedan ser incorporadas por algún software genérico que ayude a los médicos a diagnosticar. Sin embargo, algunas clínicas utilizan software propietario de análisis basados en inteligencia artificial u otras modalidades (Deserno 2011; Le et al., 2019), los cuales, en general, están diseñado sobre bases de datos de mamogramas ( Dembroker et al., 2019; Lee et al., 2017). 
Un punto importante es el valor de la fuerza de compresión que se debe aplicar en cada paciente. Existen estudios sobre la uniformidad de fuerzas y la relación con parámetros de calidad de imágenes (Korf et al., 2009; Poulos et al., 2004; Dustler et al., 2012; Hossain et al., 2015) para definir los mejores rangos, sin embargo todavía no hay definiciones sobre este tema.

\section{Desarrollo}

En la siguiente tabla se muestra el cronograma planteado para el trabajo de investigación.

\begin{tabular}{|c|c|c|c|c|c|c|c|c|c|c|c|c|c|c|c|c|c|c|c|c|}
\hline \multirow{2}{*}{ Planificación } & \multicolumn{4}{|c|}{2020} & \multicolumn{4}{|c|}{2021} & \multicolumn{4}{|c|}{2022} & \multicolumn{4}{|c|}{2023} & \multicolumn{4}{|c|}{2024} \\
\hline & Q1 & Q2 & Q3 & Q4 & Q1 & Q2 & Q3 & Q4 & Q1 & Q2 & Q3 & Q4 & Q1 & Q2 & Q3 & Q4 & Q1 & Q2 & Q3 & Q4 \\
\hline 1. Manejo y Organización & & & & & & & & & & & & & & & & & & & & \\
\hline Tarea 1.1 -Cursar materias & & & & & & & & & & & & & & & & & & & & \\
\hline Tarea 1.2 - Estado del arte & & & & & & & & & & & & & & & & & & & & \\
\hline $\begin{array}{l}\text { Tarea 1.3- Organización de trabajo } \\
\text { general }\end{array}$ & & & & & & & & & & & & & & & & & & & & \\
\hline $\begin{array}{l}\text { 2. Diseño de fantomas uniformes y } \\
\text { pruebas de los materiales }\end{array}$ & & & & & & & & & & & & & & & & & & & & \\
\hline $\begin{array}{l}\text { Tarea } 2.1 \text { Pruebas de los materiales } \\
\text { usados para construir fantomas. }\end{array}$ & & & & & & & & & & & & & & & & & & & & \\
\hline $\begin{array}{l}\text { Tarea } 2.2 \text { Diseño y desarrollo de } \\
\text { fantomas uniformes con silicona }\end{array}$ & & & & & & & & & & & & & & & & & & & & \\
\hline $\begin{array}{l}\text { Tarea } 2.3 \text { Diseño de fantomas } \\
\text { uniformes con PVAL }\end{array}$ & & & & & & & & & & & & & & & & & & & & \\
\hline $\begin{array}{l}\text { Tarea } 2.4 \text { Diseño y desarrollo de } \\
\text { fantomas uniformes con impresora } \\
\text { 3D }\end{array}$ & & & & & & & & & & & & & & & & & & & & \\
\hline $\begin{array}{l}\text { 3. Métodos computacionales para el } \\
\text { desarrollo de los fantomas simil } \\
\text { mama }\end{array}$ & & & & & & & & & & & & & & & & & & & & \\
\hline $\begin{array}{l}\text { Tarea 3.1 Trabajar con base de datos } \\
\text { de mamografía DBT }=\text { Machine } \\
\text { learning }\end{array}$ & & & & & & & & & & & & & & & & & & & & \\
\hline $\begin{array}{l}\text { Tarea 3.2: Creación de una mama } \\
\text { virtual tipo estandar con lesiones }\end{array}$ & & & & & & & & & & & & & & & & & & & & \\
\hline $\begin{array}{l}\text { Tarea 3.3: Metodologías para crear } \\
\text { fantoma 3D con impresora, PVAL, } \\
\text { silicona }\end{array}$ & & & & & & & & & & & & & & & & & & & & \\
\hline $\begin{array}{l}\text { Tarea } 3.4 \text { Capacitación en machine } \\
\text { learning y otras metodologías } \\
\text { necesarias. }\end{array}$ & & & & & & & & & & & & & & & & & & & & \\
\hline 4. Diseño de fantomas & & & & & & & & & & & & & & & & & & & & \\
\hline $\begin{array}{l}\text { Tarea 4.1: Diseño fantomas } \\
\text { antropomórficos }\end{array}$ & & & & & & & & & & & & & & & & & & & & \\
\hline $\begin{array}{l}\text { Tarea 4.2: Validación de los } \\
\text { fantomas, con base de datos y en } \\
\text { forma experimental (machine } \\
\text { learning y otros) }\end{array}$ & & & & & & & & & & & & & & & & & & & & \\
\hline 5. Aplicación de los fantomas & & & & & & & & & & & & & & & & & & & & \\
\hline $\begin{array}{l}5.1 \text { Pruebas de fuerza de compresión } \\
\text { vs calidad imagen }\end{array}$ & & & & & & & & & & & & & & & & & & & & \\
\hline
\end{tabular}




\begin{tabular}{|l|l|l|l|l|l|l|l|l|l|l|l|l|l|l|}
\hline $\begin{array}{l}5.2 \text { Pruebas de procesamiento de } \\
\text { imágenes }\end{array}$ & \\
\hline $\begin{array}{l}\text { 6. Publicaciones y escritura de Tesis } \\
\text { Tarea 5.1: Actividades relacionadas a } \\
\text { publicación y con la escritura de } \\
\text { Tesis }\end{array}$ & & &
\end{tabular}

Tabla 1: Cronograma presentado para la realización del trabajo de investigación

\section{Conclusiones}

En este trabajo se muestran los objetivos y la metodología a seguir para poder completar el trabajo de investigación en forma exitosa. Al terminar dicho trabajo se espera disponer de fantomas que simule una mama de una mujer en 3 dimensiones, de bajo costo y que se pueda realizar con reproducibilidad y exactitud para poder compartirlos con la comunidad médica-científica que lo requiera.

Se podrán realizar: capacitaciones al personal técnico/médico, actividades de investigación específicas por ejemplo respecto a los softwares usados en los mamógrafos, se podrán adquirir imágenes y analizarlas con adecuadas técnicas de procesamientos y se podrán realizar controles de calidad de imágenes en equipos tomosíntesis digital de mama de cualquier marca y modelo.

\section{Referencias}

Akki, R. S., Arunachalam, K. (2014). Breast tissue phantoms to assist compression study for cancer detection using microwave radiometry. In Engineering in Medicine and Biology Society (EMBC), 2014 36th Annual International Conference of the IEEE, 1119-1122. https://doi.org/ 10.1109/EMBC.2014.6943791.

ARTINIS: https://www.artinis.com/cdmam-34 Ultimo acceso julio 2020.

Baneva, Y. et al. (2016). Evaluation of breast software model for X-ray 2D and 3D mammography imaging. Physica Medica: European Journal of Medical Physics, 32, 255-256. https://doi.org/10.1016/j.ejmp.2017.04.024.

Bliznakova, K., Buliev, I. and Bliznakov, Z.O. (2018). Anthropomorphic Phantoms in Image Quality and Patient Dose Optimization: A EUTEMPE Network book.

Bliznakova, K., Kazakli, S., Pallikarakis, N. (2009) An Optimised 3D Breast Phantom for X-Ray Breast Imaging Techniques. In: Vander Sloten J., Verdonck P., Nyssen M., Haueisen J. (eds) 4th European Conference of the International Federation for Medical and Biological Engineering. IFMBE Proceedings, vol 22. Springer, Berlin, Heidelberg. https://doi.org/10.1007/978-3-540-89208-3_589

Brimacombre, J. M., Wilson D. R., Hodgson A. J. et al. (2009). Effect of calibration method on Tekscan sensor accuracy. Journal of biomechanical engineering, 131(3). https://doi.org/ 10.1115/1.3005165.

CIRSINC: https://www.cirsinc.com/products/ Ultimo acceso julio 2020.

Daskalaki, A. et al. (2016). Evaluation of the effect of silicone breast inserts on X-ray mammography and breast tomosynthesis images: A Monte Carlo simulation study. Physica Medica: European Journal of Medical Physics, Volume 32, Issue 2, 353 361. https://doi.org/ 10.1016/j.ejmp.2016.01.478. 
Dembrower, K., Lindholm, P. \& Strand, F. A (2020). Multi-million Mammography Image Dataset and Population-Based Screening Cohort for the Training and Evaluation of Deep Neural Networks - the Cohort of Screen-Aged Women (CSAW). J. Digit. Imaging, 33(2), 408-413. https://doi.org/ 10.1007/s10278-019-00278-0.

Deserno, T.M. (2011). Biological and Medical Physics, Biomedical Engineering, Biomedical Image Processing Ed Springer. EUREF, Protocol for the Quality Control of the Physical and Technical Aspects of Digital Breast Tomosynthesis Systems Version 1.01, June 2016.

Dustler, M., Andersson, I., Förnvik, D., and Tingberg, A. (2012). The effect of breast positioning on breast compression in mammography: a pressure distribution perspective. Proc. SPIE 8313, Medical Imaging 2012: Phys. of Med. Imag., 83134M. https://doi.org/ 10.1117/12.905746.

Feng S.S., and Sechopoulos I. (2012). Clinical digital breast tomosynthesis system: dosimetric characterization.Radiology, 263(1), 35-42. DOI: 10.1148/radiol.11111789.

Feradov F., Marinov S., Bliznakova K. (2019). Published Physical Breast Phantom Dedicated for Mammography Studies in: XV Mediterranean Conference on Medical and Biological Engineering and Computing - MEDICON

Hossain, A. et al. (2015). Study on Compression Induced Contrast in X-ray Mammograms Using Breast Mimicking Phantoms. International Journal Bioautomation, 19(3). Corpus ID: 26434602.

IARC: https://gco.iarc.fr/today/data/factsheets/populations/32-argentina-fact-sheets.pdf Ultimo acceso julio 2020.

IAEA International Agency Energy Atomic. Safety Standard for protecting people and environment. Radiation Protection and Safety of Radiation Sources:International Basic Safety Standars EC,FAO,IAEA,ILO,OECD/NEA,PAHO,NEA,UNEP,General Safety Requirements PArt 3 NO.GSR part 3. (2014).

INC: https://www.argentina.gob.ar/salud/inc Ultimo acceso julio 2020.

Kiarashi, N., Nolte, A.C., Sturgeon, G.M., Segars, W.P., Ghate S.V., Nolte, L.W., Samei, E. Lo, J.Y. (2015). Development of realistic physical breast phantoms matched to virtual breast phantoms based on human subject data. Med Phys.,42(7),41164126. https://doi.org/ 10.1118/1.4919771.

Korf, A, Herbst, C P, Rae, W. I.D. (2009). The relationship between compression force, image quality and radiation dose in mammography. Journal of Radiology 13, 486-92. https://doi.org/ 10.4102/sajr.v13i4.486

Le, E.P.V, Wang, Y., Huang, Y., Hickman, S. and Gilbert, F.J. (2019). Artificial intelligence in breast imaging. J. Clin Radiol. 2019 May;74(5), 357-366. DOI: https//doi.org/10.1016.j.crad.2019.02.006.

Lee, R., Gimenez, F., Hoogi, A. et al. (2017). A curated mammography data set for use in computer-aided detection and diagnosis research. Sci Data 4,170177. https://doi.org/ 10.1038/sdata.2017.177

NORMI PAS. User Manual NORMI PAS T42028 PTW-FREIBURG Germany. (2013) 
Poulos, A. and McClean D. (2004). The application of breast compression in mammography: a new perspective. Radiography, Volume 10, Issue 2, 131 - 137. https://doi.org/ 10.1016/j.radi.2004.02.012

Price, B., et al. (2010). An elastically compressible phantom material with mechanical and x-ray attenuation properties equivalent to breast tissue. Phys. in med. and biol., 55(4), 1177-1188. https://doi.org/ 10.4102/SAJR.V13I4.486

Schopphoven, S., Cavael, P., Bock, K., Fiebich, M., Mäder, U. (2019). Breast phantoms for 2D digital mammography with realistic anatomical structures and attenuation characteristics based on clinical images using 3D printing. Phys Med Biol. 23, 64(21), 215005. https://doi.org/ 10.1088/1361-6560/ab3f6a.

Silva, F., et al. (2011). Breast phantom with silicone implant for evaluation in conventional mammography. J. of Appl. Clin. Med. Phys., 12: 199-206. https://doi.org/ 10.1120/jacmp.v12i1.3340.

WHO: https://www.who.int/es/news-room/fact-sheets/detail/cancer Ultimo acceso julio 2020. 